\title{
Dialogs Taking into Account Experience, Emotions and Personality
}

\author{
Anne-Gwenn Bosser ${ }^{1}$, Guillaume Levieux ${ }^{1}$, Karim Sehaba ${ }^{2}$ \\ Axel Buendia ${ }^{4}$, Vincent Corruble ${ }^{2}$, Guillaume de Fondaumière ${ }^{3}$, Viviane Gal $^{1}$, \\ Stéphane Natkin ${ }^{1}$, and Nicolas Sabouret ${ }^{2}$ \\ ${ }^{1}$ Conservatoire National des Arts et Métiers, Paris, France \\ emcedric@cnam.fr \\ ${ }^{2}$ Laboratoire d'Informatique de Paris 6 \\ Université Pierre et Marie Curie - Paris 6, France \\ firstname.lastname@lip6.fr \\ ${ }^{3}$ Quantic Dream, Paris, France \\ quantic@quanticdream.com \\ ${ }^{4}$ SpirOps, Paris, France \\ firstname.lastname@spirops.com
}

\begin{abstract}
This paper describes the DEEP project (Dialogs taking into account Experience, Emotions and Personality, adapted to computer games), which started in June 2006. The aim of the project is to provide generic solutions for the integration of autonomous Non Player Characters (NPCs) in next-generation adventure games. DEEP NPCs, equipped with a personality and a believable emotional engine, will use contextbased information from the game environment and the player behavior to provide entertaining, rich and relevant dialogs.
\end{abstract}

Keywords: narrative intelligence, interactive narrative, interactive drama, narrative structures, behavior engine, agents, virtual characters, personality model, emotion model, dialog.

\section{Introduction}

In most computer games, especially in solo adventure games, the player immersion quality is directly related to the design of a believable environment. One of the most important constituent of such an environment is its population, the Non-Player Characters (NPCs). These are usually designed through script-level programming. This technique cannot afford the required variability, and can lead to boring, repetitive, or even inconsistent NPCs behavior 9 .

In this context, the DEEP project 11 aim is to provide generic solutions for the integration of realistic, autonomous, intelligent and emotional NPCs in nextgeneration adventure games. The project focuses on providing rich dialog between the player and NPCs. Our approach combines two fundamental research

\footnotetext{
${ }^{1}$ The DEEP project is funded by the French ministries of Culture and Communication (CNC), Research and Industry through the RIAM (Recherche et Innovation en Audiovisuel et Multimédia) network.
} 
directions usually seen as difficult to conciliate in academic research : the narrative approach and the autonomous agents approach.

The narrative approach relies on conceptualizing the player experience in the game and asserting the dialog always follows the narrative logic. The other research direction relies on providing an autonomous NPC architecture which allows the design of a wide variety of personalities for virtual characters. The combination of these two directions is identified as the main issue of the project.

This paper describes the scientific challenges faced in the context of the DEEP project and the adopted approaches to solve them. The paper is organized as follows : section 2 defines the DEEP architecture, sections 3 and 4 respectively focus on the narrative model and NPC model. We conclude with the open issues.

\section{DEEP Architecture}

The purpose of the DEEP architecture is to provide NPC dialogs, which are more relevant considering the player experience, and richer through various NPC personalities and an emotional model definition. The dialog engine uses the player and NPC models as a context base for generating the most suitable dialog, according to the current situation. These models are dynamically updated using informations gathered from the game environment. It provides the emotional state and knowledge of each NPC, the player context in the narrative and his expected knowledge.

The general architecture of DEEP is described in figure 1. It relies on three main components: the Drama Model, the NPC Model and the Game Controller.

The Game Controller is the game engine component which drives the entire DEEP dialog architecture as well as other aspects of the game-play. Particularly, it manages the Game State, which evolves according to the actions of the player or the NPC's behavior.

The Player Model is dynamically updated to reflect the player history in the game. It uses a light model of all the possible stories, defined during the game design, the Narrative Model.

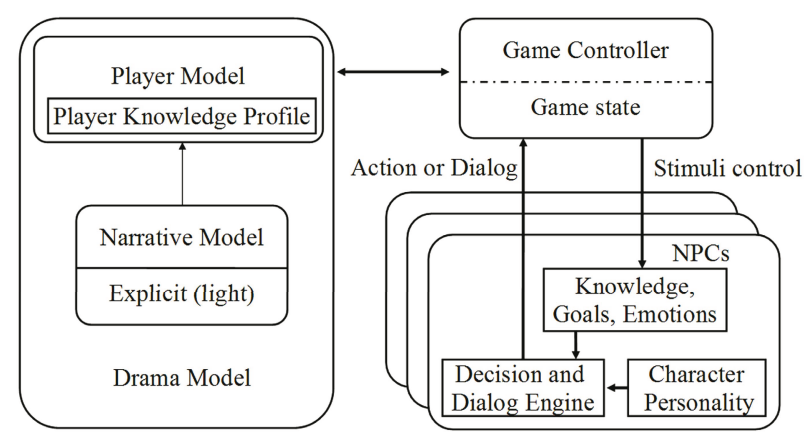

Fig. 1. General Architecture 
The Drama Model results from the narrative approach. The Game Controller uses it to update the game state, and, when necessary, to modify the NPCs knowledge, inducing specific behaviors needed for the narrative.

Events in the game are perceived as stimuli by the NPC Model. This model results from the autonomous NPC approach. It triggers a decision process in response to the stimuli, and takes into account NPCs Personality and Emotional state.

The Dialog Engine focuses on providing communication capabilities to the DEEP NPCs. It communicates with the player while selecting topics, nuancing expressions and vocabulary according to each NPC personality and emotional state.

The following two sections gives a more detailed description of the drama model and of the NPC architecture.

\section{Drama Model}

\subsection{AI in Games}

Most of the adventure game designs hypothesize on the player behavior and abilities. For instance, the level design defines, through a mix of topological and logical constraints, the player expected paths and progress in the game [6].

Using this expected behavior, the game designer anticipates a future sequence of events. In order to avoid repetitive dialogs, a NPC will then be programmed as a state automaton providing several different dialogs. This approach reflects the general "Turing test" point of view adopted in most of the games AI [13] : An intelligent behavior in a game is a behavior which seems to be intelligent considering the current knowledge of the player.

In the DEEP Project, we formalize this practical approach. We build an explicit model of the player experience, as a game designer or a scenarist would usually implicitly do. The explicit model allows the DEEP architecture to dynamically adapt to the player behavior, providing believable NPC, accurate and relevant from the player perspective.

Building a user model to improve the game design is not a new idea [1] [18] 14, but in these approaches, it is mainly the player as a human being which is modeled. In our very pragmatic approach, we design the model of the player as its avatar in the game, registering its history and experience in the game universe.

\subsection{The User Model in the DEEP Architecture}

We have identified two categories of data to represent the player history in the game, clues and environmental data, stored in the knowledge base for the user Model.

In most of the adventure games, the progress of the scenario relies on solving enigma and understanding the current plot. Information is disseminated throughout the game and clues are uncovered gradually by the player, very 
often through dialogs with NPCs. By registering correlated data, we allow the NPC dialog engine to sort out what clues the player is missing for solving the current plot.

This approach is related to Mori and Hoshino's work in which the story is controlled through key actions changing the NPC dialogs [12. However, in our approach, the dialog is modulated according to the player actions, when variation related to the game environment evolution can be introduced through the NPC emotional model (see section 4).

The model also registers environmental data, used by the dialog engine to achieve the immersion in a believable universe. These data are related to the background of the game universe, the player's avatar personal story, or the environment and atmosphere. The NPC dialog engine will use this base for picking dialog elements corresponding to the player experience, thus improving the player immersion in the game.

The user model is also linked to a light formalization of the scenario. We propose a player experience model which can be dynamically used to control the NPCs dialogs. Interactive narration models implicitly or explicitly define a partial order of events and plot points, which can be represented as a graph [6]. Our model uses goal nets [3. We associate plot points with data related to the player progress. At a given step, some of these data are known, maybe known or unknown by the player. A correct progression leads to discard some clues, hide other ones, and use known environmental data to provide realistic dialogs.

Our narrative model is locally defined by the level designer, stamping triggers, clues, environmental datas and defining a partial order between the corresponding events. Using this partial and atomic information, we are able to reconstruct an oriented graph representing the interactive story, only as exhaustive as described by the game designer. By matching this model with the dynamic description of the linear story as witnessed by the player (which is a subset of this graph) we are able to define what kind of knowledge will help the player and communicate it to him through a NPC via the DEEP dialog architecture.

\section{Emotions and Personality Model}

In this section, we explore the structure of emotions and personality information as well as its link with perception, decision and expression. Indeed, the effect of personality and emotions on behavior has been widely studied, whether it concerns a general influence on perception, behavior [10, decision-making and planning [4] or social interaction [8].

Our purpose is to introduce a NPC architecture model allowing the representation of emotions, personality and experience in order to bring flexibility and modularity in the individualized reaction of NPCs. These models must include data related to personality factors, which play an important role in differentiating physiologic and cognitive reactions of each character. They must also process emotion categories, in order to create realistic reactions of the NPC vis-a-vis specific events and to equip each one with appropriate attitudes, particularly in 
the dialog process. Processing emotions requires also to memorize the previous emotional value of events.

We first present and discuss some emotions and personality models from psychology research. Then, we briefly introduce the principles of our emotional model.

\subsection{Theoretical Bases}

Personality is an important domain of psychology research. Unfortunately, there is no consensus on the definition of personality. Thus, several personality models have been defined. These models consist of a factors set, where each factor is a specific personality property. For example, Eysenck proposes to describe personality based on extraversion and neuroticism, while the Big-five model uses five factors: extraversion, agreeableness, conscientiousness, neuroticism and openness [5] [11. In these models, each personality factor has an impact on the emotions generated from perceived events, and therefore on the character's resulting behavior. By definition, the personality factors are constant over time.

Much work, related to the Cognitive Appraisal theory, proposes various criteria in order to distinguish emotions [16] [15]. These models allow to specify properties and criteria on events which cause emotions. Roseman's model generates seventeen emotions according to a set of criteria [16]. Ortony, Clore and Collins propose an emotional model, named OCC, which cover a vast set of emotional situations [15. This model became the standard for emotions generation and is used in several works. However the emotional process defined in these models is not complete : the manner in which personality factors influences the feeling of emotions is not defined operationally. Moreover, links between emotions and their intensity, and the intensity levels corresponding to the activation of each emotion are not defined despite their importance confirmed in various experiments [7.

\subsection{Emotional Model}

Our model is based on an explicit representation of personality and emotions, and of each personality factors influence on the sensitivity to emotion categories.

The personality factors are represented by a vector $p$ of $n$ dimension and the emotional state at time $t$ by a vector $e(t)$ with $m$ dimension. $n$ and $m$ are defined according to the used models (for example, $n=5$ for Big five model, $n=2$ for Eysenck model, ...). The influence of each personality factor (let $p_{i} \in p$ ) on the sensitivity of each emotional category (let $e_{j} \in e$ ) is represented by a function $f\left(p_{i}, e_{j}\right)$. Thus, a matrix $M_{n * m}$ of functions is formed representing all influences of each personality factor on the all emotional categories. From this matrix, we can calculate the personality-emotions sensitivity. This in turn also influences the decay rate of emotions.

The model updates the emotional state according to the OCC process steps : classification, quantification, interaction and mapping [2]. These steps are started after either each time cycle or stimulus reception.

The architecture of our NPC model is a set of three components : 
- Personality and emotions: This module identifies the environment events which can affect the NPC. It evaluates their emotional potentials and calculates their influences on the emotions, taking into account the personality and the emotional memory of the NPC.

- Knowledge and reasoning: This module makes decisions adapted to the emotional state, personality and active objectives of the NPC. In the DEEP project, we focus on decisions producing dialogs.

- Behavior: This module produces emotional behaviors adapted to the situation. It consists in carrying out the knowledge and reasoning module decisions taking into account the current emotional state.

\section{Conclusion and Open Issues}

In this paper, we have described the DEEP project, and how we combine a narrative perspective with an autonomous believable agent approach in order to build an architecture providing rich and versatile NPC dialogs in adventure games. Integrating these two points of view is a challenging task, especially when designing a generic solution ready for industrial use.

The integration of emotions, personality and narratives within the NPC reasoner is the key of the DEEP project success. We are still working on this problem and have to consider several issues.

We have defined an emotional model with strong bases in psychology research. This model represents explicitly personality, emotions, and their interactions. Thus, the updating process of the emotional state is based on the personality factors of a NPC and its previous emotions modified by the last stimuli. Currently, we work on the emotional state influence on the dialog and the decision-making process.

From the narrative perspective, an interesting open issue is the way to deal with the dramatic evolution of the game, in order to extend the capabilities of the dialog engine.

\section{References}

1. Bartle, R.: Hearts, clubs, diamonds, spades: players who suit MUDs. Journal of MUD Research 1(1) (1996)

2. Bartneck, C.: Integrating the occ model of emotions in embodied characters. In: Proceedings of the Workshop on Virtual Conversational Characters: Applications, Methods, and Research Challenges, Melbourne, Australia (November 2002)

3. Cai, Y., Miao, C., Tan, A., Shen, Z.: Fuzzy cognitive goal net for interactive storytelling plot design. In: Proceedings of the ACM SIGGHI Advances in Computer Entertainment (ACE) (2006)

4. Damasio, A.R.: Lérreur de Descartes - la raison des émotions. Odile Jacob (1994)

5. Eysenck, H.J.: The biological basis of personality. In: Thomas, C.C. (ed.), Springfield, IL (1967)

6. Grunvogel, S., Natkin, S., Vega, L.: A new methodology for spatiotemporal Game Design. In: Proceedings of CGAIDE (2004) 
7. Izard, C.E., Hembree, E.A., Huebner, R.R.: Infants' emotion expressions to acute pain: Developmental change and stability of individual differences. Developmental Psychology 23, 105-113 (1987)

8. Keltner, D., Haidt, J.: Social functions of emotions. In: Mayne, T., Bonanno, G.A. (eds.) Emotions: Current issues and future directions, ch. 6, pp. 192-213. Guilford Press, New York (2001)

9. Laird, J.E., van Lent, M.: The role of ai in computer games genre. In: Handbook of computer Games study. MIT Press, Cambridge (2005)

10. Marsella, S., Gratch, J.: A step towards irrationality: Using emotion to change belief. In: Proceedings of the 1st International Joint Conference on Autonomous Agents and Multi-Agent Systems, Bologna, Italy (July 2002)

11. McCrae, R.R., John, O.P.: An introduction to the five-factor model and its applications. Journal of Personality 60, 175-215 (1992)

12. Mori, H., Hoshino, J.: Key action technique for digital storytelling. In: Kishino, F., Kitamura, Y., Kato, H., Nagata, N. (eds.) ICEC 2005. LNCS, vol. 3711. Springer, Heidelberg (2005)

13. Natkin, S.: Video Games and Interactive Media, a Glimpse at New Digital Entertainment. In: Peteres, A.K. (ed.) (2006)

14. Natkin, S., Yan, C.: User model in multiplayer mixed reality entertainment applications. In: Proceedings of the ACM SIGCHI International Conference on Advances in Computer Entertainment Technology (ACE) (2006)

15. Ortony, A., Clore, G.L., Collins, A.: The cognitive structure of emotions. Cambridge University Press, Cambridge (1988)

16. Roseman, I.J., Jose, P.E., Spindel, M.: Appraisals of emotion-eliciting events: testing a theory of discrete emotions. Journal of Personality and Social Psychology 59(5), 899-915 (1990)

17. Sehaba, K., Estraillier, P., Lambert, D.: Interactive educational games for autistic children with agent-based system. In: Kishino, F., Kitamura, Y., Kato, H., Nagata, N. (eds.) ICEC 2005. LNCS, vol. 3711, pp. 422-432. Springer, Heidelberg (2005)

18. Szilas, N.: Interactive drama on computer: beyond linear narrative. In: Proceedings of the AAAI Fall Symposium on Narrative Intelligence (1999) 